\title{
Keywords
}

computer/internet technology; empirically supported treatments; health services; internet; primary care; treatment

\begin{abstract}
Depression is the leading cause of worldwide disability and a leading contributor to global healthcare costs (WHO, 2017). Efficacious depression treatments are available, including medications and behavioral treatments, yet a variety of clinical questions influence whether a patient receives effective treatment, including: What clinical concerns should be targeted in
\end{abstract}

Corresponding author: Kevin A. Hallgren, University of Washington Medical Center 1959 NE Pacific St., Box 356560, Seattle, WA 98195, telephone: 206-616-2906, fax: 206-543-9520. 
treatment? Is a patient likely to respond to a given treatment? What are the likelihoods of side effects? Is a given treatment approach working? When it is appropriate to change the type, dose, or frequency of treatment? These are common clinical questions that all providers who treat depression routinely address, and improving providers' capacities for making informed clinical decisions around these questions will improve the effectiveness of depression treatment.

Digital technologies are growing rapidly and have the potential to shape the quality, scale, and method of depression care delivery (Clarke \& Yarborough, 2013; Mohr, Burns, Schueller, Clarke, \& Klinkman, 2013). Concurrently, healthcare systems are continuously adapting to new practice standards, scientific findings, and service and reimbursement models. As changes in technology and healthcare continue, there are growing opportunities to usher in technologies to support clinical decision-making in depression treatment. Previous studies have reviewed how digital technologies can deliver depression treatment as stand-alone interventions (e.g., computer-, website-, and mobile app-based treatment; Donker et al., 2013; Huguet et al., 2016; Newman, Szkodny, Llera, \& Przeworski, 2011); however, few studies have reviewed the ways digital technologies can support clinical decision-making.

Our goal in this paper is to provide snapshots of current research findings, near-term challenges, and opportunities for digital technologies to support clinical decision-making for providers who commonly treat depression (e.g., primary care providers, specialty mental health providers). We first provide an overview of clinical decision-making, then discuss clinical challenges and opportunities for technology to support and enhance clinical decision-making.

\section{Depression and Clinical Decision-Making}

Clinical decision-making is a core element of practice. It is a multidimensional process that involves collecting, analyzing, and integrating multiple sources of data, corroborating those data with empirical research and clinical expertise, and producing actionable decisions (Croskerry \& Nimo, 2011; Stacey, Légaré, \& Kryworuchko, 2009). It is also a goal-driven activity geared toward achieving specific and measurable outcomes, such as symptom reduction or improvements in co-occurring issues (e.g., medical, behavioral, or quality-oflife problems). Depression treatment decisions often are made in collaboration with other healthcare providers who span multiple disciplines.

Contemporary clinical decision-making models emphasize patient autonomy and shared decision-making throughout the course of treatment (Makoul \& Clayman, 2006; Elwin et al., 2012), which is associated with better treatment adherence, patient satisfaction, and clinical outcomes (Bauer, Parker, et al., 2014; Clever et al., 2006; Loh et al., 2007). However, shared decision-making can be challenging for many reasons, including a culture of providercentered clinical decision-making, limited time for patient-provider communication, differences in provider and patient goals, and a tendency for treatment goals to change over time as symptoms and circumstances change. 
In sum, clinical decision-making for depression treatment is a goal-directed process that is collaboratively formulated with patients and other professionals and based on the bestavailable information about a patient's predicted response. Three broad clinical movements can support these aspects of clinical decision-making, including measurement-based care, integrated care, and personalized medicine. Current research findings, challenges, and opportunities for digital technologies within these clinical movements will be explored in the following sections.

\section{Measurement-Based Care}

Measurement-based care (MBC) for depression refers to the systematic and routine administration, scoring, and reviewing of depression symptom rating scales to inform clinical decision-making, and this practice is a fundamental component of many treatment modalities (Fortney et al., 2016; Trivedi \& Daly, 2007). By routinely collecting standardized measures of depression severity, MBC helps providers identify response to treatment earlier than clinical judgment alone (Hatfield, McCullough, Frantz, \& Krieger, 2010), yielding larger, faster, and less costly improvements in mental health symptoms (Hawkins, Lambert, Vermeersch, Slade, \& Tuttle, 2004; Knaup, Koester, Schoefer, Becker, \& Puschner, 2009). MBC may also foster better patient-provider communication and help patients and providers navigate treatment goals and shared decision-making (Scott \& Lewis, 2016). Many practice organizations have called for systematic implementation of MBC, including the Institute of Medicine (IOM, 2006) and the American Psychiatric Association (Gelenberg et al., 2010).

Despite its clinical benefits, it is estimated that less than 1 in 5 psychiatrists and less than 1 in 9 psychologists routinely use MBC (Zimmerman \& McGlinchey, 2008; Hatfield et al., 2010). The use of MBC in mental healthcare substantially lags its use for physical health conditions, often due to practical barriers. MBC for physical health problems typically relies on biometric values reported in the electronic health record systems (EHRs), whereas MBC for depression often relies on patient-reported outcomes, which are not routinely administered or entered into EHRs (Lyon \& Lewis, 2016). Most MBC assessment tools do not measure treatment goals that are not directly reflected as symptoms of depression or other medical conditions, and most MBC tools only provide snapshots of symptoms at the time of clinical appointments and little or no information about patient outcomes between appointments. Despite consistent empirically-demonstrated benefits, many providers view MBC as burdensome and unhelpful for guiding clinical decision-making (Jensen-Doss \& Hawley, 2010). Several technological opportunities may improve the implementation of MBC for depression, including enhanced integration with other EHRs, incorporating data on treatment process and quality with $\mathrm{MBC}$ data, and leveraging the potential of mobile and biosensor data to enrich MBC.

\section{Opportunity: Integrating Patient-Reported Outcomes into Electronic Health Records}

Multiple systems exist for collecting, storing, and reviewing patient-reported outcomes for MBC, but most do not integrate with commonly-used EHRs (Lyon, Lewis, Boyd, Hendrix, \& Liu, 2016). Integrating tools that support MBC for depression with existing EHRs could 
greatly enhance the reach and efficiency of $\mathrm{MBC}$ while reducing provider burden (Gleacher et al., 2016; Steinfeld, Franklin, Mercer, Fraynt, \& Simon, 2016).

Many administrative and technological barriers exist for integrating MBC and EHRs (Lyon \& Lewis, 2016), including a limited presence of behavioral health elements in EHRs, limited interoperability and access to behavioral health data across different settings, and variability in EHRs and system requirements (Karakus, Ghose, Goldman, Moran, \& Hogan, 2017). Implementation strategies will be necessary to better integrate patient-reported outcomes into EHRs, including studying key barriers and facilitators of integration, education on the benefits of MBC for different stakeholders, and obtaining stakeholder input across multiple domains (e.g., clinical, administrative, data management, software design, business) and throughout stages of development and implementation (Gleacher et al., 2016; Lyon, Wasse, et al., 2016).

There are several research and design opportunities to enhance the quality, usability, and clinical impact of tools that support MBC and their integration into EHRs. Tools that automate or support aspects of provider workflows, or that facilitate timely and goal-directed communication between patients and providers or within healthcare teams, are likely to be more valued and therefore more successfully implemented in clinical practice. Recent research raises the concern that existing EHRs are an impediment to patient-provider communication, for example, with physicians spending up to twice as much time performing EHR-related tasks than making direct contact with patients (Sinsky et al., 2016). Shortages of time are common reasons that providers do not use MBC or shared decision-making processes (Légaré, Ratté, Gravel, \& Graham, 2008), and there are considerable research opportunities to identify ways that providers can implement MBC to enhance both workflow efficiency and patient-provider communication. Usability and user-centered design studies could inform the development of tools that work toward these aims, identifying sources of and solutions to provider frustrations (e.g., "alert fatigue" from completing multiple clinical reminders; reduced clinical time due to increasing EHR-related tasks; Ash, Sittig, Campbell, Guappone, \& Dykstra, 2007; Lyon, Wasse, et al., 2016), yielding tools that work in the service of their clinical needs without adding to their frustrations.

\section{Opportunity: Measuring Treatments Received}

A major aim of $\mathrm{MBC}$ is to gauge the effectiveness of a given treatment in facilitating improved outcomes. Linking depression symptom data with depression treatment service data can simplify clinical judgments on whether and how much an existing treatment may be affecting symptoms (Chorpita, Daleiden, \& Bernstein, 2016). Thus, there are opportunities to explore ways to integrate treatment process data with depression symptom data, and to understand how that could inform clinical decision-making.

Measuring the type, dose, and quality of treatment is itself a challenging feat with many research opportunities. For example, there are several mobile applications for tracking medication adherence (Dayer, Heldenbrand, Anderson, Gubbins, \& Martin, 2013), although these tools are not widely used by patients and are currently unlikely to integrate with many EHRs. A simple tool that tracks real-time medication-taking and summarizes this 
information with relevant alerts for providers could be a promising opportunity for digital tools to enhance MBC for depression.

Measuring the type and quality of psychotherapy is even more challenging. Psychotherapy quality can vary substantially, and providers are usually unable to assess their own level of skill or treatment adherence. Recent research has applied automated speech recognition, natural language processing, and machine learning methods to automatically and accurately assess psychotherapy quality metrics such as empathy and counselor reflections and questions within motivational interviewing (Atkins et al., 2014; Pace et al., 2016; Xiao et al., 2015, 2016). NIMH, IOM, and the Affordable Care Act explicitly support assessment of psychotherapy quality on a large scale, noting the lack of this as a rate-limiting factor for large-scale delivery of effective psychosocial treatments. Research providing automated feedback to counselors for training, supervision, and quality assurance is just beginning, and future research is needed to assess the impact of such technology on depression outcomes (Gibson et al., 2016).

\section{Opportunity: Harnessing Mobile and Biosensor Data.}

Mobile devices (e.g., smartphones) and wearable biosensors (e.g., fitness bands) have the capacity to expand the quantity, quality, and temporal accuracy of data to inform MBC by capturing data "passively", requiring minimal effort from the user and yielding data with minimal self-perception bias. Such devices can detect whether and when patients are engaging in various activities (e.g., sleeping, walking, running), physiological states (e.g., heart rate), geospatial locations, or communication patterns (e.g., texting, calling, emailing). Several small-sample studies suggest these data can predict mental health symptoms (e.g., Asselbergs et al. 2016; Ben-Zeev, Scherer, Wang, Xie, \& Campbell, 2015; Faurholt-Jepsen et al., 2016); however, the robustness of these methods continues to be established.

Smartphones also have the capacity to collect self-reported depression symptom ratings on a much more frequent basis than most current MBC practices. Tools that support this form of self-monitoring may themselves provide therapeutic benefit (Poston \& Hanson, 2010) by helping patients understand their symptoms better, and many patients report interest and willingness to use mobile devices for this (Torous, Friedman, \& Keshavan, 2014).

At the same time, it is unclear whether greater volume of depression symptom data can improve clinical decision-making or depression treatment outcomes. While patients may wish to share this type of data with their clinical providers, there are few tools that help providers access, integrate, and review the large volumes of data that can be collected through mobile devices (Chung, Cook, Bales, Zia, \& Munson, 2015; Torous \& Baker, 2016). Additional research is needed to identify methods for efficiently extracting actionable information from mobile data with minimal effort on behalf of providers, and to understand the extent to which this data impacts clinical decision-making and depression outcomes.

\section{Integrated Care}

In addition to - and often in tandem with - MBC, there is growing interest and utilization of integrated care, in which medical and mental health treatment are provided jointly, often 
with multiple providers addressing multiple clinical issues concurrently. Integrated care is a flexible approach for coordinating care that can involve multiple formats (e.g., psychotherapy, medication management) to address medical and mental health conditions. The Collaborative Care Model is one of the most well-researched integrated care models with strong empirical support for improving clinical outcomes, reducing costs, and improving access to depression treatment (Archer et al., 2012; Coventry et al., 2014). Treatment decisions in the Collaborative Care Model are managed by teams of providers from different health disciplines, which allows each team member's expertise to be matched with specific treatment-related tasks corresponding to their clinical role (e.g., psychiatric consultation, patient outreach, care management). This team-based care efficiently leverages providers' specialized expertise to larger patient populations compared to direct specialty care by independent providers (Wagner, Austin, \& Von Korff, 1996; Bower, Gilbody, Richards, Fletcher, \& Sutton, 2006). Clinical registries serve as a critical center point for integrated care coordination and planning.

Integrated care models are increasingly being adopted as scientific, practice, and reimbursement models support these models of care (e.g., value-based care, medical home models; Kazak, Nash, Hiroto, \& Kaslow, 2017). Digital technologies have the capacity to provide scaffolding that proactively supports the implementation and efficiency of integrated care team communication and coordination.

\section{Opportunity: Supporting Treatment Team Communication and Care Coordination}

Effective patient-provider and provider-provider communication are essential to the provision of integrated care. Yet, patient-provider communication has become increasingly limited in an era when a physician's office-based evaluation for depression may last fewer than 2 minutes (Tai-Seale, McGuire, Colenda, Rosen, \& Cook, 2007). Likewise, high caseloads and lack of physical co-location between medical and mental health providers limits provider-provider communication. In their seminal work describing the Chronic Care model, Wagner and colleagues (1996) outlined the key role of technological infrastructure for supporting effective interactions between patients and care teams through shared care plans and clinical decision support tools, specifically emphasizing patient registries and reminder systems for ensuring proactive and consistent follow-ups.

Web-based registries for providers have emerged as key tools for facilitating effective Collaborative Care (Unützer, Choi, Cook, \& Oishi, 2002). In parallel, electronic portals that give patients direct access to their medical records are a relatively new innovation. When integrated with provider-facing tools, such patient-facing tools have the potential to extend and streamline care coordination (Bauer, Thielke, Katon, Unützer, \& Areán, 2014) by granting a central portal for accessing and modifying information on depression symptoms and treatment. Patients and providers are generally receptive toward tools that streamline integrated care, and most primary care patients are comfortable with sharing this information with healthcare provider teams via smartphone applications (Bauer, Iles-Shih, Ghomi, Grover, \& Monsell, 2016; Bauer et al., in press; Bruns, Hyde, Sather, Hook, \& Lyon, 2016). Technologies that support communication with treatment providers outside of clinical sessions may promote patient engagement by helping patients feel connected and cared for 
by providers, even if communications are limited to standardized questionnaires to supplement brief or infrequent clinical visits. Well-designed health care portals could enhance patient engagement and provide a platform for clinical education around symptoms and treatments. There is considerable room for identifying ways to improve the effectiveness and efficiency with which providers can manage remote patient data in clinical registries and reach actionable decisions from them (Office of the National Coordinator for Health Information Technology, 2015).

\section{Personalized Medicine}

Finally, there is increasing interest in information and tools that support the delivery of mental healthcare that is tailored to individual patients, pinpointing which treatments provide the optimal benefit for which patients at the right time (e.g., Insel et al., 2010). Research suggests that individual patient characteristics can predict responses to different depression treatments (DeRubeis et al., 2014); however, developing more personalized depression treatments will likely require "big data" on clinical outcomes across large combinations depression treatment approaches, crossed with the multitude of genetic, biomedical, behavioral, environmental, and demographic patient characteristics that could predict different responses to different treatments. While randomized clinical trials (RCTs) have typically been the gold standard for testing treatment efficacy, RCTs cannot provide all the necessary data to identify optimal treatments across all patient characteristics (Trivedi, Fava, Marangell, Osser, \& Shelton, 2006). The NIH's precision medicine initiative (Precision Medicine Initiative Working Group, 2015) aims to support these efforts by enrolling one million patients into a single, large-scale study of health. Existing, large-scale datasets in EHRs may also inform these efforts.

\section{Opportunity: Supporting Discovery in Personalized Medicine}

"Big data" in EHRs could provide a useful source for informing areas where RCT data are insufficient in identifying who is more likely to respond to different treatments and at which times (Kessler et al., 2017). EHR databases can provide valuable, real-world, practice-based evidence to support better models of predicting which patients are at risk for a specific disorder or outcome (e.g., suicide; McCarthy et al., 2015) or which treatments may be most effective for a particular patient. The ability to alert providers to conduct targeted screens (e.g., for suicidality) and consider delivering personalized interventions (e.g., via electronic clinical decision-support tools for addressing suicide), may be particularly appealing in primary care, where providers often have limited capacity to conduct numerous clinical screens or personalize depression treatments during brief appointments (Kurian et al., 2004).

\section{Conclusion}

Changes in healthcare and technology are increasing the opportunities to design, test, and implement tools that can support more effective and efficient clinical decision-making. The opportunities discussed here focus on using technology to provide "scaffolding" to support clinical decision-making approaches with already-established empirical support (e.g., MBC, integrated care). Several of these opportunities are summarized in Table 1.

Depress Anxiety. Author manuscript; available in PMC 2018 September 14. 
Successfully integrating clinical, scientific, and technological frameworks to support clinical decision-making will require substantial efforts in clinical science, technology design, data science, implementation science, and other fields. Utilizing implementation strategies (e.g., stakeholder engagement) throughout the process of developing and testing these technologies will likely facilitate more successful downstream implementation (Bruns et al., 2016).

It is important to note that clinical decision-making is a broad and complex process. There are undoubtedly additional aspects of clinical decision-making that may be improved through technology but were not discussed here, and additional technological opportunities will continue to grow. For example, micro-randomized clinical trials and just-in-time adaptive interventions are two emerging methodologies that hold promise for enhancing fine-grained treatment decisions to deliver more optimal treatments that are patient-tailored and timed for optimal effectiveness (Ben-Zeev et al., 2014; Nahum-Shani et al., 2015).

Technology can rapidly revolutionize the ways we communicate and access information; for example, the iPhone was first released in 2007 and personal communication has evolved drastically over the decade since that time. In the coming decade, it is possible that thoughtfully designed, clinically-integrated technologies can similarly help usher in innovations in clinical decision-making for depression and other mental illnesses.

\section{Acknowledgements:}

This work was supported by National Institute of Alcoholism and Alcohol Abuse grant numbers K01AA024796 and K02AA023814, and by National Center for Advancing Translational Sciences grant number KL2TR000421. The content is solely the responsibility of the authors and does not necessarily represent the official views of the National Institutes of Health. Drs. Hallgren and Bauer report no conflicts of interest. Dr. Atkins has received grant funding from Microsoft and is a health science advisor for Behavioral Informatix, a start-up focused on developing technologies for behavioral health.

\section{References}

Archer J, Bower P, Gilbody S, Lovell K, Richards D, Gask L, Dickens C, \& Coventry P (2012). Collaborative care for depression and anxiety problems. Cochrane Database of Systematic Reviews 2012, Issue 10. Art. No.: CD006525. doi: 10.1002/14651858.CD006525.pub2.

Ash JS, Sittig DF, Campbell EM, Guappone KP, \& Dykstra RH (2007). Some unintended consequences of clinical decision support systems. AMIA Annual Symposium Proceedings, 2007, 26-30.

Asselbergs J, Ruwaard J, Ejdys M, Schrader N, Sijbrandij M, \& Riper H (2016). Mobile phone-based unobtrusive ecological momentary assessment of day-to-day mood: An explorative study. Journal of Medical Internet Research, 18(3), e72. [PubMed: 27025287]

Atkins DC, Steyvers M, Imel ZE, \& Smyth P (2014). Scaling up the evaluation of psychotherapy: evaluating motivational interviewing fidelity via statistical text classification. Implementation Science, 9, 49. [PubMed: 24758152]

Bauer AM, Iles-Shih M, Ghomi RH, Grover T, Monsell S (8, 2016) Augmenting Collaborative Care through mobile health: A pilot study Presentation at the $23^{\text {rd }}$ NIMH Conference on Mental Health Services Research; August 2, 2016; Washington DC.

Bauer AM, Parker MM, Schillinger D, Katon W, Adler N, ... Karter AJ (2014). Associations between antidepressant adherence and shared decision-making, patient-provider trust, and communication among adults with diabetes: Diabetes study of Northern California (DISTANCE). Journal of General Internal Medicine, 29(8), 1139-1147. [PubMed: 24706097] 
Bauer AM, Rue T, Munson SA, et al. (in press). Patient-oriented health technologies: Patients' perspectives and use. Journal of Mobile Technology in Medicine.

Bauer AM, Thielke SM, Katon W, Unützer J, Areán P (2014). Aligning health information technologies with effective service delivery models to improve chronic disease care. Preventive Medicine, 66, 167-172. [PubMed: 24963895]

Ben-Zeev D, Scherer EA, Wang R, Xie H, \& Campbell AT (2015). Next-generation psychiatric assessment: Using smartphone sensors to monitor behavior and mental health. Psychiatric Rehabilitation Journal, 38(3), 218-226. [PubMed: 25844912]

Ben-Zeev D, Brenner CJ, Begale M, Duffecy J, Mohr DC, \& Mueser KT (2014). Feasibility, acceptability, and preliminary efficacy of a smartphone intervention for schizophrenia. Schizophrenia Bulletin, 40(6), 1244-1253. [PubMed: 24609454]

Bower P, Gilbody S, Richards D, Fletcher D, \& Sutton A (2006). Collaborative care for depression in primary care: Making sense of a complex intervention: Systematic review and meta-regression." British Journal of Psychiatry, 189(6): 484-493. doi:10.1192/bjp.bp.106.023655. [PubMed: 17139031]

Bruns EJ, Hyde KL, Sather A, Hook AN, \& Lyon AR (2016). Applying user input to the design and testing of an electronic behavioral health information system for wraparound care coordination. Administration and Policy in Mental Health and Mental Health Services Research, 43, 350-368. [PubMed: 26060099]

Clarke G, \& Yarborough BJ (2013). Evaluating the promise of health IT to enhance/expand the reach of mental health services. General Hospital Psychiatry, 35(4), 339-344. [PubMed: 23701698]

Clever SL, Ford DE, Rubenstein LV, Rost KM, Meredith LS, Sherbourne CD, ... Cooper LA (2006). Primary care patients' involvement in decision-making is associated with improvement in depression. Medical Care, 44(5), 398-405. [PubMed: 16641657]

Chorpita BF, Daleiden EL, \& Bernstein AD (2016). At the intersection of health information technology and decision support: Measurement feedback systems... and beyond. Administration and Policy in Mental Health and Mental Health Services Research, 43, 471-477. [PubMed: 26604202]

Chung CF, Cook J, Bales E, Zia J, \& Munson SA (2015). More than telemonitoring: Health provider use and nonuse of life-log data in irritable bowel syndrome and weight management. Journal of Medical Internet Research, 17(8), e203. [PubMed: 26297627]

Coventry PA, Hudson JL, Kontopantelis E, Archer J, Richards DA, ... Bower P (2014). Characteristics of effective collaborative care for treatment of depression: A systematic review and metaregression of 74 randomised controlled trials. PLoS One, 9(9), e108114. [PubMed: 25264616]

Croskerry P, \& Nimmo GR (2011). Better clinical decision making and reducing diagnostic error. Journal of the Royal College of Physicians of Edenborough, 41, 155-162.

Dayer L, Heldenbrand S, Anderson P, Gubbins PO, \& Martin BC (2013). Smartphone medication adherence apps: Potential benefits to patients and providers. Journal of the American Pharmacists Association, 53(2), 172-181. [PubMed: 23571625]

DeRubeis RJ, Cohen ZD, Forand NR, Fournier JC, Gelfand LA, \& Lorenzo-Luaces L (2014). The personalized advantage index: Translating research on prediction into individualized treatment recommendations. A demonstration. PLoS One, 9(1), e83875. [PubMed: 24416178]

Donker T, Petrie K, Proudfoot J, Clarke J, Birch MR, \& Christensen H (2013).Smartphones for smarter delivery of mental health programs: A systematic review. Journal of Medical Internet Research, 15(11), e247. [PubMed: 24240579]

Elwin G, Frosch D, Thomson R, Joseph-Williams N, Lloyed A, Kinnersley P, ... Barry M (2012). Shared decision making: A model for clinical practice. Journal of General Internal Medicine, 27(10), 1361-1367. [PubMed: 22618581]

Faurholt-Jepsen M, Vinberg M, Frost M, Debel S, Margrethe Christensen E, Bardran JE, \& Kessing LV (2016). Behavioral activities collected through smartphones and the association with illness activity in bipolar disorder. International Journal of Methods in Psychiatric Research, 25(4), 309323. [PubMed: 27038019] 
Fortney JC, Unützer J, Wrenn G, Pyne JM, Smith GR, Schoenbaum M, \& Harbin HT (2016). A tipping point for measurement-based care. Psychiatric Services, epub ahead of print. 10.1176/ appi.ps.201500439

Gelenberg AJ, Freeman MP, Markowitz JC, Rosenbaum JF, Thase ME, Trivedi MH, \& Van Rhoads RS (2010). Practice guideline for the treatment of patients with major depressive disorder (3rd ed.). Washington, DC: American Psychiatric Association.

Gibson J, Gray G, Hirsch T, Imel ZE, Narayanan SS, \& Atkins DC (2016b, 5 8). Developing an Automated Report Card for Addiction Counseling: The Counselor Observer Ratings Expert for MI (CORE-MI) Presented at CHI '16, CHI Conference on Human Factors in Computing Systems, Computing and Mental Health Workshop, San Jose, CA.

Gleacher AA, Olin SS, Nadeem E, Pollock M, Ringle V, Bickman L, Douglas S, \& Hoagwood K (2016). Implementing a measurement feedback system in community mental health clinics. A case study of multilevel barriers and facilitators. Administration and Policy in Mental Health and Mental Health Services Research, 43, 426-440. [PubMed: 25735619]

Hatfield D, McCullough L, Frantz SH, \& Krieger K (2010). Do we know when our clients get worse? An investigation of therapists' ability to detect negative client change. Clinical Psychology and Psychotherapy, 17(1), 25-32. [PubMed: 19916162]

Hawkins EJ, Lambert MJ, Vermeersch DA, Slade KL, \& Tuttle KC (2004). The therapeutic effects of providing patient progress information to therapists and patients. Psychotherapy Research, 14(3), 308-327.

Huguet A, Rao S, McGrath PJ, Wozney L, Wheaton M, Conrod J, \& Rozario S (2016) A systematic review of cognitive behavioral therapy and behavioral activation apps for depression. PLoS One, 11(5): e0154248. doi: 10.1371/journal.pone.0154248 [PubMed: 27135410]

Insel T, Cuthbert B, Garvey M, Heinssen R, Pine DS, Quinn K, Sanislow C, \& Wang P (2010). Research domain criteria (RDoC): Toward a new classification framework for research on mental disorders. The American Journal of Psychiatry, 167(7), 748-751. [PubMed: 20595427]

Institute of Medicine. (2006). Improving the quality of health care for mental and substance use conditions: Quality chasm series. Washington, DC: The National Academies Press.

Jensen-Doss A, \& Hawley KM (2010). Understanding barriers to evidence-based assessment Clinician attitudes toward standardized assessment tools. Journal of Clinical Adolescent Psychology, 39(6), 885-896.

Karakus M, Ghose SS, Goldman HH, Moran G, \& Hogan MF (2017). "Big eight" recommendations for improving the effectiveness of the U.S. behavioral health care system. Psychiatric Services, 68(3), 288-290. [PubMed: 27524367]

Kazak AE, Nash JM, Hiroto K, \& Kaslow NJ (2017). Psychologists in patient-centered medical homes (PCMHs): Roles, evidence, opportunities, and challenges. American Psychologist, 72(1), 1-12. [PubMed: 28068134]

Kessler RC, van Loo HM, Wardenaar KJ, Bossarte RM, Brenner LA, Ebert DD, ... Zaslavsky AM (2017). Using patient self-reports to study heterogeneity of treatment effects in major depressive disorder. Epidemiology and Psychiatric Sciences, 26(1), 22-36. [PubMed: 26810628]

Knaup C, Koester M, Schoefer D, Becker T, \& Puschner B (2009). Effect of feedback of treatment outcome in specialist mental healthcare: meta-analysis. The British Journal of Psychiatry, 195(1), 15-22. [PubMed: 19567889]

Kurian BT, Trivedi MH, Grannemann BD, Claassen CA, Daly EJ, \& Sunderajan P (2004). A computerized decision support system for depression in primary care. Primary Care Companion to the Journal of Clinical Psychiatry, 11(4), 140-146.

Légaré F, Ratté S, Gravel K, \& Graham ID (2008). Barriers and facilitators to implementing shared decision-making in clinical practice: Update of a systematic review of health professionals' perceptions. Patient Education and Counseling, 73(3), 526-534. [PubMed: 18752915]

Loh A, Simon D, Wills CE, Kriston L, Niebling W, Härter M (2007). The effects of a shared decisionmaking intervention in primary care of depression: A cluster randomized controlled trial. Patient Education and Counseling, 67(3), 324-332. [PubMed: 17509808]

Lyon AR \& Lewis CC (2016). Designing health information technologies for uptake:Development and implementation of measurement feedback systems in mental health service delivery. 
Administration and Policy in Mental Health and Mental Health Services Research, 43, 344-349. [PubMed: 26658691]

Lyon AR, Lewis CC, Boyd MR, Hendrix E, \& Liu F (2016). Capabilities and characteristics of digital measurement feedback systems: Results from a comprehensive review. Administration and Policy in Mental Health and Mental Health Services Research, 43, 441-466. [PubMed: 26860952]

Lyon AR, Wasse JK, Ludwig K, Zachry M, Bruns EJ, Unutzer J, \& McCauley E (2016). The contextualized technology adaptation process (CTAP): Optimizing health information technology to improve mental health systems. Administration and Policy in Mental Health and Mental Health Services Research, 43, 394-409. [PubMed: 25677251]

Makoul G, \& Clayman ML (2006). An integrative model of shared decision making in medical encounters. Patient Education and Counseling, 60, 301-312. [PubMed: 16051459]

Marcus M, Yasamy MT, van Ommeren M, \& Chisholm D (2012). Depression: A global public health concern. Retrieved from http://www.who.int/mental_health/management/depression/ who_paper_depression_wfmh_2012.pdf

McCarthy JF, Bossarte RM, Katz IR, Thompson C, Kemp J, Hannemann CM, Nielson C, \& Schoenbaum M (2015). Predictive modeling and concentration of the risk of suicide: Implications for preventive interventions in the US Department of Veterans Affairs. American Journal of Public Health, 105(9), 1935-1942. [PubMed: 26066914]

Mohr DC, Burns MN, Schueller SM, Clarke G, \& Klinkman M (2013). Behavioral intervention technologies: Evidence review and recommendations for future research in mental health. General Hospital Psychiatry, 35(4), 332-338. [PubMed: 23664503]

Nahum-Shani S, Smith SN, Tewari A, Witkiewitz K, Collins LM, Spring B, \& Murphy SA (2014). Just-in-time adaptive interventions (JITAIs): An organizing framework for ongoing health behavior support. (Technical Report No. 14-126). University Park, PA: The Methodology Center, Penn State.

Newman MG, Szkodny LE, Llera SJ, \& Przeworski A, (2011). A review of technology-assisted selfhelp and minimal contact therapies for anxiety and depression: Is human contact necessary for therapeutic efficacy. Clinical Pyschology Review, 31(1), 89-103.

Office of the National Coordinator for Health Information Technology (2015). Federal health IT strategic plan, 2015-2020. Office of the Secretary, United States Department of Health and Human Services Retrieved from https://www.healthit.gov/sites/default/files/9-5federalhealthitstratplanfinal_0.pdf

Pace B, Tanana M, Xiao B, Dembe A, Soma C, Steyvers M, Narayanan SS, Atkins DC, \& Imel ZE (2016). What about the words? Natural language processing in psychotherapy. Psychotherapy Bulletin, 51(1), 17-18.

Precision Medicine Initiative Working Group (2015). The Precision Medicine Initiative Cohort Program - Building a Research Foundation for $21^{\text {st }}$ Century Medicine. National Institutes of Health Retrieved from https://www.nih.gov/sites/default/files/research-training/initiatives/pmi/ pmi-working-group-report-20150917-2.pdf

Poston JM, \& Hanson WE (2010). Meta-analysis of psychological assessment as a therapeutic intervention. Psychological Assessment, 22(2), 203-212. [PubMed: 20528048]

Scott K, \& Lewis CC (2016). Using measurement-based care to enhance any treatment. Cognitive Behavioral Practice, 221), 49-59.

Sinsky C, Colligan L, Li L, Prgomet M, Reynolds S, Goeder L, ... Blike G (2016). Allocation of physician time in ambulatory practice: A time and motion study in 4 specialties. Annals of Internal Medicine, 165(11), 753-760. [PubMed: 27595430]

Stacey D, Légaré F, \& Kryworuchko J, (2009). Evidence-based health care decision-making: Roles for health professionals In Edwards A \& Elwyn G, Shared Decision-Making in Health Care: Achieving Evidence-Based Patient Choice ( $2^{\text {nd }}$ ed.). New York: Oxford. ${ }^{\text {nd }}$

Steinfeld B, Franklin A, Mercer B, Fraynt R, \& Simon G (2016). Progress monitoring in an integrated health care system: Tracking behavioral health vital signs. Administration and Policy in Mental Health and Mental Health Services Research, 43, 369-378. [PubMed: 25840521] 
Tai-Seale M, McGuire T, Colenda C, Rosen D, Cook MA (2007) Two-minute mental health care for elderly patients: Inside primary care visits. Journal of the American Geriatric Society, 55(12), 1903-1911.

Torous J, \& Baker JT (2016). Why psychiatry needs data science and data science needs psychiatry: Connecting with technology. JAMA Psychiatry, 73(1), 3-4. [PubMed: 26676879]

Torous J, Friedman R, \& Keshavan M (2014). Smartphone ownership and interest in mobile applications to monitor syptoms of mental health conditions. Journal of Medical Internet Research, MHealth and UHealth, 2(1), e2.

Trivedi MH, \& Daly EJ (2007). Measurement-based care for refractory depression: A clinical decision support model for clinical research and practice. Drug and Alcohol Dependence, 88S, S61-S71.

Trivedi MH, Fava M, Marangell LB, Osser DN, \& Shelton RC (2006). Use of treatment algorithms for depression. Primary Care Companion to the Journal of Clinical Psychiatry, 8(5), 291-298.

Unützer J, Choi Y, Cook IA, \& Oishi S (2002). A web-based data management system to improve care for depression in a multicenter clinical trial. Psychiatric Services, 53(6),671-678. [PubMed: 12045303]

Wagner EH, Austin BT, \& Von Korff M (1996). Organizing care for patients with chronic illness. The Milbank Quarterly, 74(4): 511-544. [PubMed: 8941260]

Xiao B, Huang C, Imel ZE, Atkins DC, Georgiou P, \& Narayanan SS (2016). A technology prototype system for rating therapist empathy from audio recordings in addiction counseling. PeerJ Computer Science, 2, e59. doi: 10.7717/peerj-cs.59.

Xiao B, Imel ZE, Georgiou PG, Atkins DC, \& Narayanan SS (2015). "Rate My Therapist": Automated Detection of Empathy in Drug and Alcohol Counseling via Speech and Language Processing. PLoS One, 10(12), e0143055 url:10.1371/journal.pone.0143055. [PubMed: 26630392]

Zimmerman M \& McGlinchey JB, (2008). Why don't psychiatrists use scales to measure outcome when treating depressed patients? Journal of Clinical Psychiatry, 69, 1916-1919. [PubMed: 19192467] 
Table 1

Example clinical decision-making questions and supporting technologies in depression treatment.

\begin{tabular}{ll}
\hline Measurement-Based Care & \\
\hline $\begin{array}{l}\text { Is my patient getting better with their } \\
\text { current treatment regimen, or should I } \\
\text { change the treatment? }\end{array}$ & $\begin{array}{l}\text { Current Technology. Standardized depression rating scales are completed at time of clinical sessions, } \\
\text { possibly entered into electronic health record systems, and possibly graphed or summarized to } \\
\text { monitor treatment progress. }\end{array}$ \\
& $\begin{array}{l}\text { Potential Technology. Behavioral and psychological indicators of depression are assessed remotely } \\
\text { through standardized rating scales and passive mobile and biosensor data. Patient-reported outcomes } \\
\text { are integrated with other data on treatment service delivery and quality (e.g., treatments administered, } \\
\text { treatment fidelity, patient adherence) with graphical or summary feedback to help assess impact of } \\
\text { treatment on patient outcomes. }\end{array}$ \\
\hline
\end{tabular}

\section{Collaborative Care}

How do I know which patients are "falling through the cracks"?
Current Technology: Providers develop and maintain a patient registry with all patients in need of depression care. The registry includes data on patient visits, outreach efforts, and clinical outcome measures.

Patients who are not engaged in care are flagged, triggering reminders for clinicians to provide outreach.

Potential Technology: A patient registry is automatically populated by the electronic health record and patient-generated data. An algorithm identifies patients who are not engaged in care or not responding to treatment, triggering automated outreach based on patients' preference for communication (e.g., primary language, and preferred contact method, such as interactive voice response, SMS, or mobile app). Providers are alerted when automated efforts are unsuccessful and additional clinical action is needed. Providers are notified in advance if the patient visits primary care or another service (e.g., dental service, nutrition, etc.) and can attempt an in-person outreach to re-engage the patient in depression care.

How can patients and provider teams communicate effectively and efficiently to facilitate care coordination?
Current Technology: Providers access clinical registries that track patients' depression data and care plans. Medical and mental health data may be integrated and accessible to all treatment providers, or in some settings mental health records may be stored separately and accessible only to a limited set of providers. Patient outreach and communication are usually handled through face-to-face contact, telephone outreach, and electronic messaging.

Potential Technology: Patients and provider teams access shared electronic health record system where patients and all team members easily access and review depression data (e.g., via clinic computers or mobile devices). Patients and provider teams may use this system to coordinate and edit treatment goals and plans, address questions, and provide education with the goal of enhancing patient and team engagement in decision-making, often remotely and between clinical sessions.

\section{Precision Medicine}

How can I identify which of my patients are at risk for serious clinical events (e.g., psychotic episode, suicide attempt) and select an appropriate intervention strategy?
Current Technology: Electronic reminders prompt providers to screen patients for specific issues (e.g., suicide risk), either universally or in response to other individual data points (e.g., responses in other screening tools, patient diagnoses). Clinical note templates or order sets may help structure and document a corresponding treatment plan.

Potential Technology: Prediction algorithms identify levels of risk for whole patient populations based on all electronic health record and patient-generated data and suggest appropriate, possibly patientmatched, interventions. Patient registry data are likewise used to help improve future iterations of prediction algorithms that can help identify clinical risks in the same or other healthcare settings.

Note. Current technologies reflect designs of some existing technologies for supporting clinical decision-making; however, there is considerable variability in whether and how such tools are implemented and designed in practice. Potential technologies reflect ideas for design improvements that could help support more effective and efficient clinical decision-making. 